\title{
Derivation of equations to define inflection point and its analysis in flattening filter free photon beams based on the principle of polynomial function
}

\author{
K R Muralidhar \\ Department of Radiation Physics, American Oncology Institute, Hyderabad, India
}

Received August 08, 2014; Revised October 29, 2014; Accepted October 29, 2014; Published Online December 05, 2014

\section{Original Article}

\begin{abstract}
Purpose: The objective of this work is to (1) present a mechanism for calculating inflection points on profiles at various depths and field sizes, and (2) study the doses at the inflection points for various field sizes at depth of maximum dose (Dmax) for flattening filter free (FFF) photon beam profiles. Methods: Graphical representation was done on percentage of dose versus inflection points. Also, using the polynomial function, the author formulated equations for calculating spot-on inflection point on the profiles for both the $6 \mathrm{MV}$ and $10 \mathrm{MV}$ energies for different field sizes at various depths. Results: In a $10 \mathrm{MV}$ FFF radiation beam, the dose at inflection point of the profile decreases as the field size increases. However, in 6MV FFF radiation beam, the dose at the inflection point initially increases with an increase in the field size up to $10 \times 10 \mathrm{~cm}^{2}$ and decreases after $10 \times 10 \mathrm{~cm}$. The polynomial function was fitted for both the $6 \mathrm{MV}$ and $10 \mathrm{MV}$ FFF beams for all field sizes and depths. Conclusion: Polynomial function is one of the easiest ways of identifying the inflection point in FFF beam for various field sizes and depths. Graphical representation of dose versus inflection point for both FFF energies was derived.
\end{abstract}

Keywords: Flattening Filter Free Beam; Inflection Point; Polynomial Function

\section{Introduction}

In conventional linear accelerators, a flat photon beam is produced with a flattening filter to simplify treatment planning in radiotherapy by delivering homogeneous dose distribution. In latest radiotherapy technologies such as intensity-modulated radiosurgery (IMRS), stereotactic body radiotherapy (SBRT) and gated treatments, treatment time is prolonged. By removing the flattening filter, treatment time can be reduced due to increment in dose rate. ${ }^{1} \mathrm{~A}$ number of studies have analysed and reported the flattening filter free (FFF) beam characteristics.

Standard parameters to define symmetry, flatness, and penumbra for a flattened beam (FB) are different from a FFF beam since FB is a forward peaked beam with reduced scattering. Many cancer centers use the parameters from the American Association of Physicists in Medicine (AAPM) Task Group 142 for the quality assurance of flat photon beams. ${ }^{2}$ Parameters such as beam flatness, symmetry, and penumbra may not be directly useful for the new FFF beams. Therefore, there is a need to find new parameters, which can be used for both the standard FF and FFF radiation beams. This can be explained with the concept of an "inflection point" (IP).
An inflection point is defined as the mid-point on either side of the high gradient region (sharply descending part) of the FFF beam profile. From the inflection point, it is possible to evaluate penumbra and the field size. A challenge in FFF beam profile is the assessment of location of inflection point. It is complicated by general beam characteristics ${ }^{3-7}, \mathrm{FFF}$ mode, forward peaked beam, the large variations of flatness and central axis positions, beam energy and depth doses ${ }^{8-11}$, back scatter ${ }^{12}$, and electron contamination. ${ }^{13}$ The ultimate goal of inflection point evaluation is location of inflection point for FFF beam with various field sizes at different depths for $6 \mathrm{MV}$ (hereafter referred as 6X) and $10 \mathrm{MV}$ (hereafter referred as 10X). To date, an accurate and computationally efficient solution is far from reach, and simplified evaluation is frequently performed in a clinical environment.

Hence, it is very important and necessary to find the accurate value of inflection point, because

- It is located in the region of highest gradient.

- In this region, the variation of dose is in the order of $10 \% / \mathrm{mm}$.

- To date, there is no fine measurement stepping and detector size for the accurate measurement of this point. 
- $\quad$ Dose can be affected by $10 \%$. It can also influence the central axis dose by up to $40 \%$ in FFF beam due to normalization at the inflection point. ${ }^{14}$ Literature for a mathematical model or formula to find the inflection point is limited.

The main purpose of this study is to develop a formula to identify an inflection point on any FFF beam profile curve for $6 \mathrm{X}$ and 10X photon beams for field sizes ranging from $1 \times$ $1 \mathrm{~cm}^{2}$ to $40 \times 40 \mathrm{~cm}^{2}$ at any depth, and this will take into account percentage of doses at inflection point versus field size. At the heart of the framework is a mechanism for calculating inflection point on FFF beam profile in both in-line and cross-line. Furthermore, detailed analysis of doses at inflection points versus field sizes for $6 \mathrm{X}$ and $10 \mathrm{X}$ FFF photon beams is presented in this paper.

\section{Methods and Materials}

\section{Relation between Percentage depth dose versus Field size in FFF beams}

6X FFF and 10X FFF photon beams were selected for this study. The relationship between the FFF beam percentages of dose values at Dose Maximum $\left(\mathrm{D}_{\max }\right)$ versus field size was analysed. Beam data were taken with the help of Blue phantom 2 Radiation Field Analyser (RFA) (IBA Dosimetry $\mathrm{GmbH}$, Germany) with CC13 and CC01 ionization chambers (IBA Dosimetry GmbH, Germany). Profiles were taken from field sizes $1 \times 1 \mathrm{~cm}^{2}$ to $40 \times 40 \mathrm{~cm}^{2}$ at depths from $D_{\max }$ to 30 $\mathrm{cm}$. All profiles were normalized at $D_{\max }$ for analysis with the help of Omnipro -Accept software (version 7.1) (IBA Dosimetry $\mathrm{GmbH}$, Germany). In-line and cross-line profiles were analysed individually for both the energies. Graphs comparing percentage of doses at inflection point versus field sizes were plotted for both the energies in in-line and cross-line directions. Figures 1 to 4 show the relationship between the percentages of doses at inflection point versus field size for $6 \mathrm{X}$ FFF in-line, $6 \mathrm{X}$ FFF cross-line, 10X FFF in-line, and 10X FFF cross-line profiles at $D_{\max }$.

\section{Inflection point calculation on $6 \mathrm{X}$ FFF and 10X FFF profiles in both inline and cross-line}

The field size in FFF is defined using inflection points, which is defined as the mid-point on either side of the high gradient region (sharply descending part) of the beam profile. Alternatively, as suggested by Pönisch et al. ${ }^{5}$ the distance between the left and right inflection points could be used to define field size. For the start point (S) and end point (E) in high gradient region of the beam profile, the separation between $\mathrm{S}$ and $\mathrm{E}$ is the height $(\mathrm{h})$ of the high gradient region of the beam profile. IP is located at $\mathrm{h} / 2$ from either location ( $\mathrm{S}$ or E). ${ }^{5}$

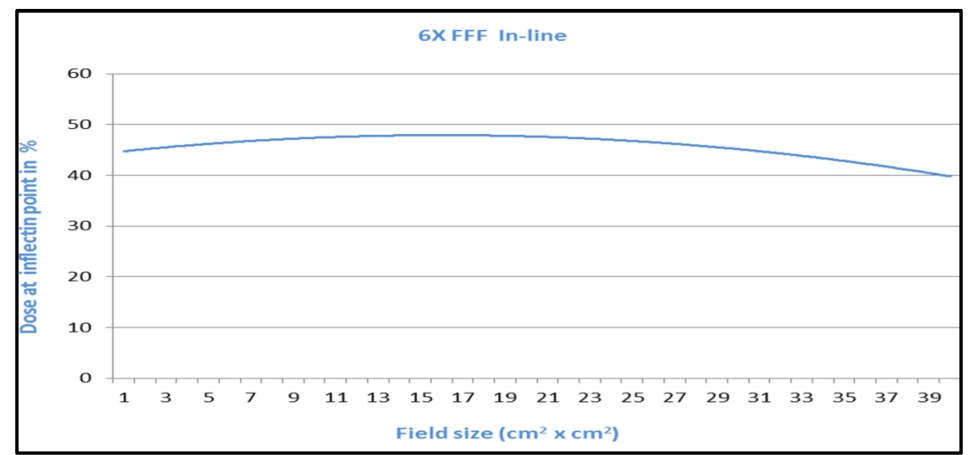

FIG. 1: Percentage of doses at inflection point in $6 \mathrm{X}$ FFF in-line profiles for field sizes up to $40 \times 40 \mathrm{~cm}^{2}$.

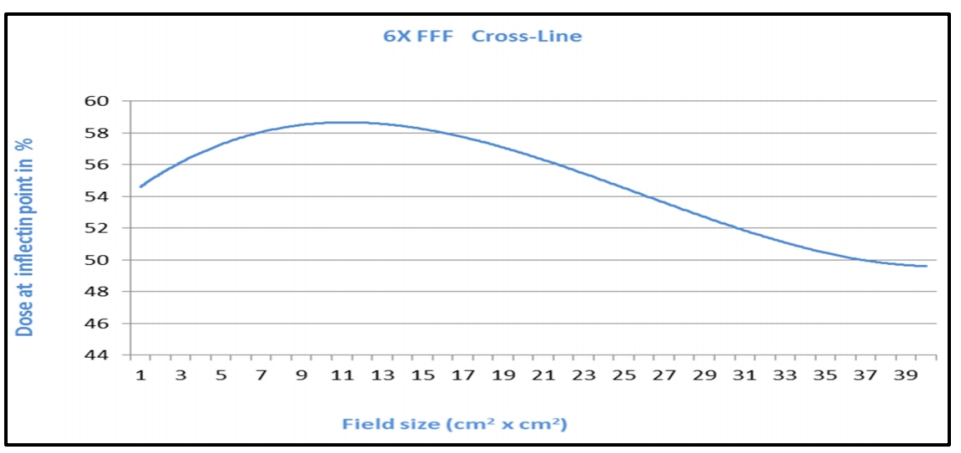

FIG. 2: Percentage of doses at inflection point in 6X FFF cross-line profiles for field sizes up to $40 \times 40 \mathrm{~cm}^{2}$.

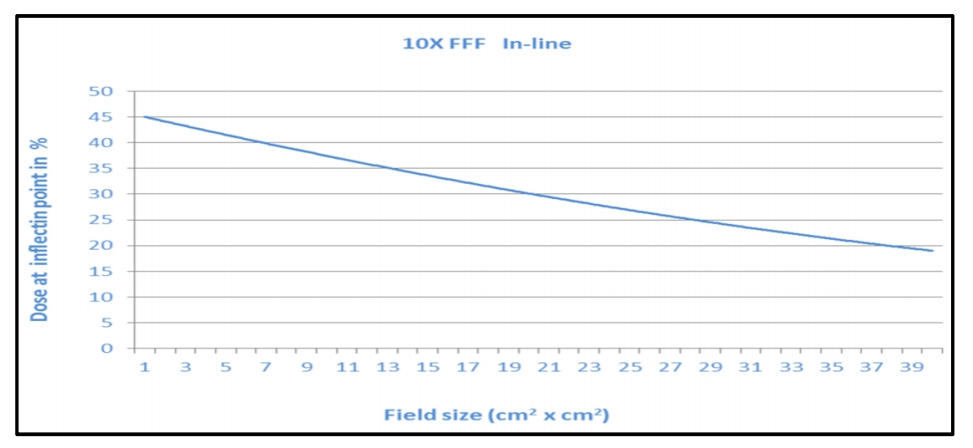

FIG. 3: Percentage of doses at inflection point in 10X FFF in-line profiles for field sizes up to $40 \times 40 \mathrm{~cm}^{2}$.

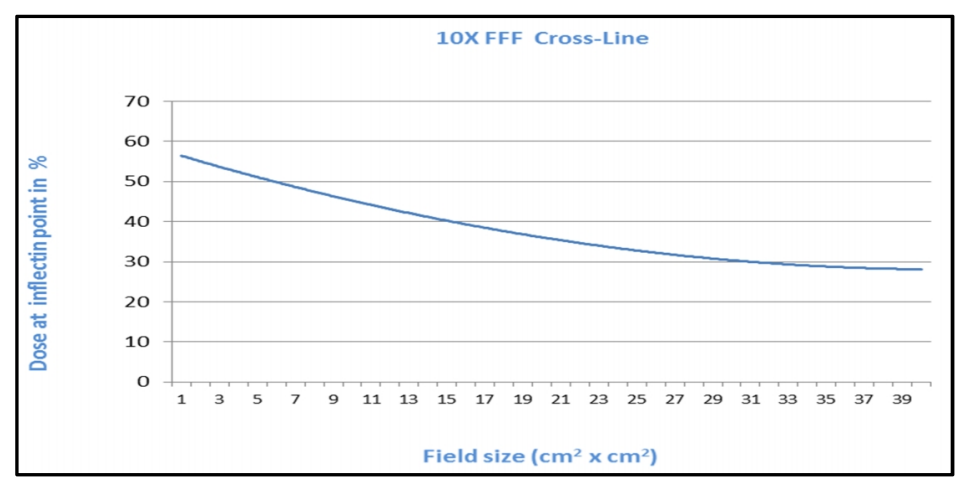

FIG. 4: Percentage of doses at inflection point in 10X FFF cross-line profiles for field sizes up to $40 \times 40 \mathrm{~cm}^{2}$. 
In the present study, using the dose at the inflection point (IP) verses field size at $D_{\max }$, we formulated equations for computing location of the inflection point on any field size and depth in $6 \mathrm{X}$ and 10X FFF beam profiles. First, the graphs comparing doses at inflection point versus field size for both energies at various depths were plotted. These inflection points were derived for each profile from the method which was explained in the above paragraph. Based on the principle of polynomial equation, the inflection point on FFF curve is computed using the trend line option for curves. All fields were normalized at $\mathrm{D}_{\max }$ to $100 \%$. Second, readings were then analysed at various depths. Based on the percentage of doses at off axis, depth dependent factors were derived. Polynomial functions are a class of functions having many important properties. They are all continuous, smooth, entire, and computable.

For 6X FFF inline and cross-line, 10X FFF Inline and cross-line, polynomial equation derived was

$\mathrm{y}=\left[-0.0141 \mathrm{x}^{2}+0.451 \mathrm{x}+44.299\right]{ }^{*} \mathrm{DDF}-\mathrm{---------------} \mathrm{Eq.} 1$

with $\mathrm{R}^{2}=0.9926$

$\mathrm{y}=\left[0.0007 \mathrm{x}^{3}-0.0557 \mathrm{x}^{2}+0.978 \mathrm{x}+53.697\right]{ }^{*} \mathrm{DDF}------$ Eq. 2 with $\mathrm{R}^{2}=0.9921$

$\mathrm{y}=\left[0.0058 \mathrm{x}^{2}-0.9062 \mathrm{x}+45.944\right]^{*} \mathrm{DDF}-----------------$ Eq. 3

with $\mathrm{R}^{2}=0.9928$

$\mathrm{y}=\left[0.0039 \mathrm{x}^{2}-1.0824 \mathrm{x}+56.499\right]^{*} \mathrm{DDF}-----------------$ Eq. 4

with $\mathrm{R}^{2}=0.9939$

where, $\mathrm{y}=$ percentage depth dose at inflection point, $\mathrm{x}=$ field size, $\mathrm{R}^{2}=\mathrm{R}$-Squared value, and $\mathrm{DDF}=$ depth dependent factor [see Table 1].

The DDF is derived from the 6X FFF, 10X FFF inline and cross line profiles. All data was normalized at Dmax. Hence, there is no change in dose up to $5 \mathrm{~cm}$ depth. From $5 \mathrm{~cm}$ to 10 $\mathrm{cm}$ depth, $1 \%$ increase in dose was observed for both in-line and cross-line for $6 \mathrm{X} \mathrm{FFF}$, whereas for $10 \mathrm{X} \mathrm{FFF}, 1 \%$ decrease in dose was observed. Similarly, data were evaluated for depths from 10 to $15 \mathrm{~cm}, 15$ to $20 \mathrm{~cm}$, and above $20 \mathrm{~cm}$ depth in Table 1. These factors are suitable for all field sizes at any depth for both the energies.

\section{Procedure to obtain the depth dependent factors}

1. FFF profiles were obtained using blue phantom 2 RFA with 0.13CC ionization chamber.

2. All PDDS and profiles were analysed using Omnipro software V7.1

3. Off axis dose was measured for all field sizes at various depths and presented in Table 1 (a) to Table 1(d). These values were normalized at $D_{\max }$ and shown as Table 1 (depth dependent factors).
4. Polynomial equations were derived from the graph between dose at inflection point verses field size was already shown in section $B$.

5. Combination of (multiplication) these two (3 and 4) gives us the factor " $y$ ", which is percentage depth dose at inflection point.

TABLE 1: Depth dependent factors to derive inflection points at various depths for 6X FFF and 10X FFF photon beams.

\begin{tabular}{cccccc}
\hline \hline & \multicolumn{5}{c}{ DDF } \\
\cline { 2 - 6 } Profile & \multicolumn{5}{c}{ Depth $(\mathbf{c m})$} \\
\cline { 2 - 6 } & $<\mathbf{5 c m}$ & $\mathbf{5}$ to $\mathbf{1 0}$ & $\mathbf{1 0}$ to $\mathbf{1 5}$ & $\mathbf{1 5}$ to $\mathbf{2 0}$ & $>\mathbf{2 0}$ \\
\cline { 2 - 6 } 6X-In-line & 1 & 1.01 & 1.05 & 1.09 & 1.12 \\
6X-Cross-line & 1 & 1.01 & 1.03 & 1.04 & 1.05 \\
10X-In-line & 1 & 0.9 & 0.9 & 0.9 & 0.9 \\
10X-Cross-line & 1 & 0.9 & 0.9 & 0.9 & 0.9 \\
\hline \hline
\end{tabular}

Abbreviations: FFF= Flattening Filter Free; DDF= Depth dependent factors

TABLE 1(a): Dose measured at off axis in FFF beam profiles at various depths at field size distance from central axis for $6 \mathrm{X}$ Cross-line profile

\begin{tabular}{llllll}
\hline \hline \multirow{2}{*}{ FS } & \multicolumn{5}{c}{ Depth (cm) } \\
\cline { 2 - 6 } & Dmax & 5 & 10 & 20 & 30 \\
\hline $4 \times 4$ & $52.73 \%$ & $53.3 \%$ & $54.04 \%$ & $54.86 \%$ & $55.57 \%$ \\
$10 \times 10$ & $51.27 \%$ & $51.41 \%$ & $51.91 \%$ & $53.22 \%$ & $54.22 \%$ \\
$15 \times 15$ & $48.38 \%$ & $48.79 \%$ & $48.7 \%$ & $49.93 \%$ & $51.3 \%$ \\
$20 \times 20$ & $44.76 \%$ & $44.96 \%$ & $44.98 \%$ & $46.07 \%$ & $47.52 \%$ \\
$30 \times 30$ & $38.42 \%$ & $37.94 \%$ & $38.37 \%$ & $39.11 \%$ & $39.85 \%$ \\
\hline \hline
\end{tabular}

Abbreviation: FS = Field Size $(\mathrm{cm} \times \mathrm{cm})$; Dmax $=$ Depth of maximum dose

TABLE 1(b): Dose measured at off axis in FFF beam profiles at various depths at field size distance from central axis for $6 \mathrm{X}$ in-line profile

\begin{tabular}{lccccc}
\hline \hline \multirow{2}{*}{ FS } & \multicolumn{5}{c}{ Depth (cm) } \\
\cline { 2 - 6 } & Dmax & $\mathbf{5}$ & \multicolumn{1}{c}{10} & $\mathbf{2 0}$ & 30 \\
\hline $4 \times 4$ & $52.73 \%$ & $53.35 \%$ & $54.04 \%$ & $54.87 \%$ & $55.61 \%$ \\
$10 \times 10$ & $39.88 \%$ & $40.34 \%$ & $41.77 \%$ & $43.63 \%$ & $44.7 \%$ \\
$15 \times 15$ & $38.54 \%$ & $39.08 \%$ & $39.47 \%$ & $41.33 \%$ & $42.62 \%$ \\
$20 \times 20$ & $35.24 \%$ & $36.12 \%$ & $37.16 \%$ & $38.67 \%$ & $40.1 \%$ \\
$30 \times 30$ & $31.47 \%$ & $31.48 \%$ & $33.36 \%$ & $34.5 \%$ & $35.33 \%$ \\
\hline \hline
\end{tabular}

Abbreviation: $\mathrm{FS}=$ Field Size $(\mathrm{cm} \times \mathrm{cm})$; Dmax $=$ Depth of maximum dose

\section{Results and Discussion}

\section{Relation between percentage of dose versus field size in FFF beam}

The Figures 1-4 shows that the dose at inflection point for $6 \mathrm{X}$ FFF beam increases as the field size increase from $1 \times 1$ $\mathrm{cm}^{2}$ to $15 \times 15 \mathrm{~cm}^{2}$ in in-line and up to $12 \times 12 \mathrm{~cm}^{2}$ in 
cross-line. Beginning from the field sizes $\left(15 \times 15 \mathrm{~cm}^{2}\right.$ in in-line and $12 \times 12 \mathrm{~cm}^{2}$ in cross-line) to the maximum field size $\left(40 \times 40 \mathrm{~cm}^{2}\right)$, continuous decrement in dose at inflection point was observed. However, in 10X, the dose at inflection point decreases continuously as the field size increases.

TABLE 1(c): Dose measured at off axis in FFF beam profiles at various depths at field size distance from central axis for $10 \mathrm{X}$ in-line profile

\begin{tabular}{llllll}
\hline \hline \multirow{2}{*}{ FS } & \multicolumn{5}{c}{ Depth (cm) } \\
\cline { 2 - 6 } & Dmax & $\mathbf{5}$ & \multicolumn{1}{c}{10} & $\mathbf{2 0}$ & 30 \\
\hline $4 \times 4$ & $44.35 \%$ & $43.01 \%$ & $43.5 \%$ & $44.02 \%$ & $44.3 \%$ \\
$10 \times 10$ & $40.96 \%$ & $36.72 \%$ & $37.69 \%$ & $38.95 \%$ & $39.56 \%$ \\
$15 \times 15$ & $38.76 \%$ & $33.51 \%$ & $33.57 \%$ & $34.54 \%$ & $35.55 \%$ \\
$20 \times 20$ & $35.37 \%$ & $29.78 \%$ & $30.35 \%$ & $31.33 \%$ & $32.51 \%$ \\
$30 \times 30$ & $32.57 \%$ & $24.25 \%$ & $25.91 \%$ & $27.12 \%$ & $26.95 \%$ \\
\hline \hline
\end{tabular}

Abbreviation: FS = Field Size $(\mathrm{cm} \times \mathrm{cm})$; Dmax $=$ Depth of maximum dose

TABLE 1(d): Dose measured at off axis in FFF beam profiles at various depths at field size distance from central axis for $10 \mathrm{X}$ cross-line profile

\begin{tabular}{llcccl}
\hline \hline & \multicolumn{5}{c}{ Depth (cm) } \\
\cline { 2 - 6 } FS & Dmax & $\mathbf{5}$ & 10 & $\mathbf{2 0}$ & 30 \\
\hline $4 \times 4$ & $53.72 \%$ & $51.7 \%$ & $52.35 \%$ & $52.62 \%$ & $52.92 \%$ \\
$10 \times 10$ & $50.25 \%$ & $45.7 \%$ & $46.2 \%$ & $47.05 \%$ & $47.59 \%$ \\
$15 \times 15$ & $46.39 \%$ & $40.87 \%$ & $41.09 \%$ & $41.88 \%$ & $42.69 \%$ \\
$20 \times 20$ & $42.43 \%$ & $35.88 \%$ & $36.13 \%$ & $37.02 \%$ & $37.76 \%$ \\
$30 \times 30$ & $36.45 \%$ & $28.63 \%$ & $28.47 \%$ & $29.31 \%$ & $30.03 \%$ \\
\hline \hline
\end{tabular}

Abbreviation: FS = Field Size $(\mathrm{cm} \times \mathrm{cm})$; Dmax = Depth of maximum dose

For both the energies, the shapes of the curves are different. In fact, the shape of the curves in $6 \mathrm{MV}$ is different between in-line and cross-line as well. The reason behind the differences of these curves are energy spectrum, scatter dose, lateral photon fluence fall off. As each energy having its own energy spectrum and all other said characters which influenced the shape of the curves. Reduction in head scatter and electron contamination is also contributing to the difference in the shape of these curves.

The in-line direction is more sensitive to the presence or absence of the scattered photons originating from $y$-jaws, because the $y$-jaws are closest to the filter location. In $6 X$, the presence of scatter is more in-line compared with $10 \mathrm{X}$ due to its properties and energy spectrum.

Irrespective of field size, in 6XFFF, the maximum values of $\%$ depth dose at inflection point were $48.05 \%$ and $59.12 \%$ in-inline and cross-line, respectively. In 10X FFF, these values were $51.37 \%$ and $57.17 \%$ in-inline and cross-line, respectively. This is very interesting new relationship, which is presented in Figures 1 to 4 . The minimum values were 39.73
$\%$ in (in-line) and $49.61 \%$ in (cross-line) for 6X FFF and $18.56 \%$ in (in-line) and $28.8 \%$ in (cross-line) for $10 \mathrm{X} \mathrm{FFF}$.

Inflection point calculation on $6 \mathrm{X}$ FFF and $10 \mathrm{X}$ FFF profiles in both in-line and cross-line

From Figure 1, the reference dose of inflection point at a given location inside profile is

$\mathrm{y}=\left[-0.0141 \mathrm{x}^{2}+0.451 \mathrm{x}+44.299\right] \times \mathrm{DDF}$

Example 1: To find the inflection point for $18 \times 18 \mathrm{~cm}^{2}$ field at depth $10 \mathrm{~cm}$ for $6 \mathrm{X}$ FFF in-line profile:

$\mathrm{Y}=\left[-0.0141 * 18 * 18+0.451^{*} 18+44.299\right]^{*} 1.01$

$\mathrm{Y}=[-4.568+8.118+44.299]^{*} 1.01$

$\mathrm{Y}=48.32 \%$.

Example 2: To find the inflection point for $22 \times 22 \mathrm{~cm}^{2}$ field at Dmax for 10XFFF cross-line profile:

$\mathrm{y}=\left[0.0039 \mathrm{x}^{2}-1.0824 \mathrm{x}+56.499\right]^{*} \mathrm{DDF}$

$\mathrm{y}=[0.0039 * 22 * 22-1.084 * 22+56.499]^{*} 1$

$\mathrm{y}=[1.8876-23.848+56.499]$

$\mathrm{y}=33.53 \%$.

The result for the inflection point in above two examples (48.32\% and $33.53 \%$ ) is essentially same as that derived by Ponisch et al. 5 (about $48.5 \%$ and $33.5 \%$ ). The author of this paper presented formula derived values are easy and accurate compared to the results obtained by expecting start and end of dose decrement and increment points. Also, this is a convenient procedure which can be implemented easily in clinic to find the inflection point in FFF. For small field sizes less than $5 \times 5 \mathrm{~cm}^{2}$, the inflection point and Full width at Half Maximum (FWHM) are almost the same, and hence analysis for FFF beams can be done just like in FB beams. In FFF, a change in $10 \%$ of dose at inflection point can impact the field width by $1 \mathrm{~mm}^{5}$ and the central axis dose level could vary up to $40 \%$ due to the normalization to the inflection point. ${ }^{14}$

In this study, we revealed a connection between the percentage of the dose at the inflection point to the field size and depth. We extended the applications of $\mathrm{Y}$ to calculate the inflection point for any field size and depth for both energies. In statistics, the coefficient of determination is denoted by $\mathrm{R}^{2}$ and the pronounced $\mathrm{R}$ squared indicates how well data points fit a line or curve. It is a statistic used in the context of statistical models whose main purpose is either the prediction of future outcomes or the testing of hypotheses, on the basis of other related information. It provides a measure of how well observed outcomes are replicated by the model, as the proportion of total variation of outcomes explained by the model. ${ }^{15}$ The shape of the curve was observed different between in-line and cross-line. This may be due to in-plane direction is more sensitive to the presence or absence of the scattered photons originating from the $\mathrm{Y}$ jaws, which are closest to the filter location. 
This analysis was done with polynomial equation and found the solution to derive the inflection point in FFF beams. Pönisch et al. ${ }^{3}$ suggested the use of the inflection point at the field edge by renormalizing a FFF beam to the same dose level of a FF beam. It may be the easiest way to identify the position of inflection point by plotting dose difference of two adjacent measuring points but it can cause error in dose up to $10 \%$. This is because the off-axis position of the minimum or maximum \% of dose at the field edge represents the inflection point. Due to its presence in the highest gradient region and is of the order of $10 \% / \mathrm{mm}$, it can cause $10 \%$ error in dose. Hence, we need more precise and accurate procedures to analyse the field width and inflection points in FFF beams. A tool to identify the accurate location of the inflection point is must instead of predicting something on an average of two dose points.

In this study, the limitation was found to be less than $1 \%$ error in dose or $0.1 \mathrm{~mm}$ difference in field width in contrast to $10 \%$ shown in other authors, and the formula presented in this study proved to be more suitable for all depths, field sizes in both energies. Single machine data is another limitation of this study. In the near future, we expect to collect more data from different machines, and this will help in reaching the final conclusion for the formula. The main aim of this paper was to show the procedure to derive the inflection point more accurately. Future studies need to include more data obtained with different types of detectors and chambers.

\section{Conclusion}

The derivative of equations based on the polynomial equation to define inflection point concept can be used to derive the inflection point dose on a FFF beam profile at a given depth with less than $1 \%$ error in dose. The preliminary results presented in this study must be validated using data from multiple machines. Corrections can be done in future studies based on the multiple number of machine data.

\section{Conflict of interest}

The author declares that he has no conflicts of interest. The author alone is responsible for the content and writing of the paper.

\section{References}

1. Rout BK, Muralidhar KR, Ali M, et al. Dosimetric study of RapidArc plans with flattened beam (FB) and flattening filter-free (FFF) beam for localized prostate cancer based on physical indices. Int $J$ Cancer Ther Oncol 2014; 2:1-9.

2. Klein EE, Hanley J, Bayouth J, et al. Task Group 142, American Association of Physicists in Medicine. Task Group 142 report: quality assurance of medical accelerators. Med Phys 2009; 36:4197-212.

3. Cashmore J. The characterization of unflattened photon beams from a $6 \mathrm{MV}$ linear accelerator. Phys Med Biol 2008; 53: 1933-46.

4. Kragl G, af Wetterstedt S, Knäusl B, et al. Dosimetric characteristics of 6 and $10 \mathrm{MV}$ unflattened photon beams. Radiother Oncol 2009; 93:141-6.

5. Pönisch F, Titt U, Vassiliev ON, et al. Properties of unflattened photon beams shaped by a multileaf collimator. Med Phys 2006; 33:1738-46.

6. Hrbacek J, Lang S, Klöck S. Commissioning of photon beams of a flattening filter-free linear accelerator and the accuracy of beam modeling using an anisotropic analytical algorithm. Int J Radiat Oncol Biol Phys 2011; 80:1228-37.

7. Georg D, Kragl G, Wetterstedt Sa, et al. Photon beam quality variations of a flattening filter free linear accelerator. Med Phys 2010; 37:49-53.

8. Xiong G, Rogers DW. Relationship between \%dd (10)x and stopping-power ratios for flattening filter free accelerators: a Monte Carlo study. Med Phys 2008; 35:2104-9.

9. Sauer OA. Determination of the quality index $(\mathrm{Q})$ for photon beams at arbitrary field sizes. Med Phys 2009; 36:4168-72.

10. Ceberg C, Johnsson S, Lind M, Knöös T. Prediction of stopping-power ratios in flattening-filter free beams. Med Phys 2010; 37:1164-8.

11. Wang Y, Khan MK, Ting JY, Easterling SB. Surface dose investigation of the flattening filter-free photon beams. Int J Radiat Oncol Biol Phys 2012; 83: e281-5.

12. Titt U, Vassiliev ON, Pönisch F, et al. Monte Carlo study of backscatter in a flattening filter free clinical accelerator. Med Phys 2006; 33:3270-3.

13. Mesbahi A. A Monte Carlo study on neutron and electron contamination of an unflattened 18-MV photon beam. Appl Radiat Isot 2009; 67:55-60.

14. Fogliata A, Garcia R, Knöös T, et al. Definition of parameters for quality assurance of flattening filter free (FFF) photon beams in radiation therapy. Med Phys 2012; 39: 6455-64.

15. Carpenter RG. Principles and procedures of statistics, with special reference to the biological sciences. The Eugenics Review 1960; 52:172-3. 